Original paper

\title{
Brain-derived neurotrophic factor as a potential diagnostic marker in minimal hepatic encephalopathy
}

Agnieszka Stawicka', Magdalena Świderska², Justyna Zbrzeźniak', Natalia Sołowianowicz' ${ }^{1}$ Aleksandra Woszczenko', Robert Flisiak', Jerzy Jaroszewicz ${ }^{1,3}$

'Department of Infectious Diseases and Hepatology, Medical University of Bialystok, Bialystok, Poland

2Department of Physiology, Medical University of Bialystok, Bialystok, Poland

${ }^{3}$ Department of Infectious Diseases and Hepatology, Medical University of Silesia, Katowice, Poland

\begin{abstract}
Introduction: Minimal hepatic encephalopathy (MHE) is a common complication of liver cirrhosis not only leading to a decrease in the quality of life, but also predicting development of overt encephalopathy. The diagnosis of MHE usually relies on a combination of neuropsychological tests, while robust serum biomarkers are lacking. We aimed to assess serum concentrations of brain-derived neurotrophic factor (BDNF) in MHE patients.

Material and methods: Serum BDNF was assessed in 78 patients with liver cirrhosis (53 male, median age 55 years) and 40 healthy individuals. 43 subjects underwent extensive evaluation for MHE by psychometric hepatic encephalopathy score (PHES) and inhibitory control test (ICT) or critical flicker frequency (CFF).

Results: Serum BDNF was twofold lower in liver cirrhosis compared to healthy subjects [13.6 (7.8-22.6) vs. $33.0(24.1-40.7) \mathrm{ng} / \mathrm{ml}, p<0.001]$ and its decrease reflected a degree of liver insufficiency assessed by model for end-stage liver disease (MELD). BDNF showed a negative correlation with bilirubin $(R=-0.35, p=0.005)$ and international normalized ratio (INR) $(R=-0.37, p=0.003)$, and positive with platelets (PLT) $(R=0.36$, $p=0.004$ ), while no associations with age, sex, body mass index (BMI), waist-hip ratio (WHR), creatinine and ammonia were noted. Importantly, subjects with a diagnosis of MHE by at least two modalities showed the lowest levels of BDNF [10.9 (2.5-14.4) vs. $19.9(9.3-29.4) \mathrm{ng} / \mathrm{ml}, p<0.01]$. Patients with self-reported sleep disturbances had significantly lower serum BDNF [13.0 (2.5-23.4) vs. $20.0(8.4-31.3) \mathrm{ng} / \mathrm{ml}, p=0.04]$.

Conclusions: The lowest serum BDNF concentration was noted in patients with MHE and sleep disturbances, which suggests a role in pathophysiology of hepatic encephalopathy but also as a potential biomarker.
\end{abstract}

Key words: minimal hepatic encephalopathy, liver cirrhosis, BDNF, PHES, ICT.

Address for correspondence:

Agnieszka Stawicka, MD, Department of Infectious Diseases and Hepatology, Medical University of Bialystok, 14 Żurawia St., 15-540 Białystok, Poland, e-mail: stawicka_a@wp.pl

\section{Introduction}

Cirrhosis as the final stage of many chronic liver diseases is not only associated with the loss of liver function, but also significantly impacts the central nervous system (CNS) [1-4]. Patients suffer from depression, anxiety and neurocognitive disorders [3]. Liver inflammatory processes result in reduction of the number of active hepatocytes, impairment of liver detoxification processes, and consequently, increased concentration of toxins in blood, including neurotoxins: ammonia, fatty acids, phenols and mercaptans (thiols) [5, 6]. Raised levels of toxins in peripheral blood cause their increased penetration through the blood-brain barrier and accumulation in the CNS [7]. One of the neuropsychiatric and cognitive syndromes caused by liver diseases and accompanying damage to the CNS is hepatic encephalopathy (HE) [8]. HE not only reduces the quality of patients' lives, but also increases the mortality rate in patients with cirrhosis [7]. 
These patients are previously diagnosed with minimal hepatic encephalopathy (MHE), which is a state of minor psychometric abnormalities [9]. MHE, in contrast to overt HE, does not have obvious symptoms. Patients with MHE have neurological and mental manifestations such as short-term memory disorders, and impaired speed of reaction, visual perception and concentration. These symptoms are not confirmed in routine physical examination [10-12]. The influence of MHE on driving skills has been documented in numerous studies. Patients show a much higher percentage of offences and road accidents compared to healthy people $[13,14]$. The emotional sphere and social adaptation are also big problems. MHE negatively affects the quality of the patient's life, and is also associated with a greater risk of developing overt $\mathrm{HE}$ and higher mortality $[15,16]$.

The pathophysiology of HE and MHE is not fully understood. Patients may develop increased levels of blood ammonia, cerebral oedema, as well as inflammation and increased oxidative stress at different stages of the disease. Therefore, the diagnosis of MHE is very important and may support the prevention of explicit HE $[5,17]$. MHE diagnosis has an impact on further treatment and prognosis. The presence of symptoms of $\mathrm{HE}$ in the form of cognitive dysfunction is attributed to cerebral oedema. The swelling of astrocytes is triggered by the accumulation of glutamine in the astrocytes, caused by the high concentration of ammonia, and also due to the oxidative/nitrosative stress response and response to antioxidant defence systems in the brain [18]. In the process of brain ageing as well as in neurodegenerative diseases, neurotransmission and repetition functions are disturbed. Brain-derived neurotrophic factor (BDNF) has been shown to induce morphological changes mediated by receptor tyrosine kinase $\mathrm{B}$ (TrkBT) in astrocytes at active synapses, suggesting the stabilization of synaptic connections. Therefore, it can be speculated that ammonia-induced astrocyte ageing is associated with impaired synaptic stability and connectivity by lowering BDNF levels as well as blocking TrkBT-dependent BDNF signalling [17].

This communication is possible due to the released growth factor and signal molecules, including BDNF $[5,6]$. It has been observed that high expression of mature BDNF is responsible for the synaptic plasticity of the CNS $[19,20]$. BDNF may also influence the processes connected with the neurogenesis of dendrites and growth of axons. Moreover, the factor plays an important role in the development of serotonergic synapse pathways, glial cells and neurons in the hippocampus, as well as the cerebral cortex [17, 19-21]. The expression of BDNF in the hippocampus and mature cerebral cortex has been demonstrated to positively influence synaptic activity and long-term potentiation, i.e. the process responsible for learning. BDNF is secreted as a consequence of neuronal stimulation and affects the release of dopamine and glutamate from hippocampus cells [22, 23].

Brain-derived neurotrophic factor is crucial for the development of the nervous system and boosts dopaminergic activity, synaptic function and neurogenesis [7-9]. All these functions make the changes connected with the distribution of this neurotrophin non-specific and thus they are observed in numerous mental and neurological disorders. Studies based on murine models have revealed that even a small reduction in BDNF can lead to decreased synaptic plasticity within the hippocampus [22]. Compared to healthy subjects, the brains of patients with mental disorders, including schizophrenia and bipolar disorder, have shown reduced expression of BDNF. We aimed to assess serum concentrations of BDNF in liver cirrhosis with special emphasis on minimal HE.

\section{Material and methods}

\section{Patients}

Seventy-eight Caucasian patients [Polish population, 53 men, median age 55 (46-60) years] diagnosed with cirrhosis of various aetiology (alcohol, viral and autoimmune) were included in this single-centre, cross-sectional study and had serum BDNF measurement performed. Alcoholic aetiology of hepatic damage dominated in the study group - 37 (53\%) patients, hepatitis $\mathrm{B}$ virus (HBV) or hepatitis $\mathrm{C}$ virus (HCV) - $21(27 \%)$ and 20 (25\%) patients with autoimmune diseases. Diagnosis of liver cirrhosis was based on a typical clinical picture, liver biopsy, or elastography in selected cases. Exclusion criteria of the study were the presence of neurological and psychiatric diseases, as well as active alcohol abuse (in the last 3 months), the use of psychoactive substances and drugs, as well as the presence of overt encephalopathy. Furthermore, patients included in the study were negative for type 1 diabetes, human immunodeficiency virus (HIV) infection, and were not being treated with hepatotoxic drugs. Overt encephalopathy and significant neurologic comorbidities were excluded by anamnesis, neurologic examination and the mini mental state examination (MMSE) test. All subjects included were able to read and write, without motor or visual disturbances. Overt encephalopathy was excluded by performing the MMSE test - a score below 23 was the cut-off point for cognitive impairment. The clinical characteristics of the studied population are presented in Table 1 . 
Table 1. Clinical characteristics of patients and comparison of patients with and without minimal hepatic encephalopathy (data presented as median $25-75 \% \mathrm{Cl}$ )

\begin{tabular}{|c|c|c|c|c|c|c|c|c|c|c|}
\hline \multirow[t]{2}{*}{ Parameter } & \multicolumn{3}{|c|}{$\begin{array}{c}\text { Cirrhosis group } \\
n=78\end{array}$} & \multicolumn{3}{|c|}{$\begin{array}{c}\text { MHE (-) } \\
n=29\end{array}$} & \multicolumn{3}{|c|}{$\begin{array}{c}\text { MHE }(+) \\
n=14\end{array}$} & \multirow[t]{2}{*}{$P$-value } \\
\hline & Median & $25 \% \mathrm{Cl}$ & $75 \% \mathrm{Cl}$ & Median & $25 \% \mathrm{Cl}$ & $75 \% \mathrm{Cl}$ & Median & $25 \% \mathrm{Cl}$ & $75 \% \mathrm{Cl}$ & \\
\hline $\operatorname{Sex}(M / F)$ & $53 / 25$ & - & - & $18 / 11$ & - & - & $10 / 4$ & - & - & 0.73 \\
\hline Age (years) & 55 & 46 & 60 & 57 & 46 & 60 & 54 & 42 & 66 & 0.94 \\
\hline WBC $\left({ }^{*} 10^{9} / I\right)$ & 5.82 & 3.65 & 9.47 & 5.35 & 3.53 & 6.67 & 4.87 & 3.25 & 13.1 & 0.72 \\
\hline $\mathrm{HGB}(\mathrm{g} / \mathrm{dl})$ & 12.3 & 11 & 13.7 & 12.0 & 11.0 & 14.3 & 11.8 & 10.6 & 13.6 & 0.60 \\
\hline $\mathrm{PLT}\left({ }^{*} 10^{9} / \mathrm{l}\right)$ & 91 & 56.5 & 152.5 & 90 & 52 & 164 & 90 & 38 & 127 & 0.48 \\
\hline ALT (IU/I) & 44 & 29.5 & 82.5 & 34 & 22 & 72 & 55 & 27 & 73 & 0.51 \\
\hline AST (IU/I) & 76.5 & 55 & 134 & 64 & 35 & 121 & 81 & 49 & 141 & 0.39 \\
\hline Bilirubin (mg/dl) & 1.9 & 1.0 & 5.4 & 1.2 & 1.0 & 2.5 & 3.0 & 0.9 & 6.8 & 0.19 \\
\hline Creatinine (mg/dl) & 0.94 & 0.72 & 1.00 & 1.00 & 0.95 & 1.00 & 0.96 & 0.70 & 1.00 & 0.14 \\
\hline INR & 1.26 & 1.1 & 1.51 & 1.17 & 1.10 & 1.34 & 1.34 & 1.20 & 1.52 & 0.04 \\
\hline Albumin (mg/dl) & 3.2 & 2.7 & 3.5 & 3.5 & 3.1 & 3.7 & 2.7 & 2.6 & 3.2 & 0.006 \\
\hline MELD & 10.7 & 7.3 & 14.3 & 10.1 & 7.5 & 13.4 & 11.6 & 6.7 & 17.3 & 0.46 \\
\hline Serum ammonia ( $\mu \mathrm{g} / \mathrm{dl})$ & 118 & 90 & 159 & 106 & 94 & 152 & 98 & 80 & 144 & 0.47 \\
\hline D-dimers (mg/l) & 1.08 & 0.72 & 4.24 & 0.93 & 0.72 & 2.83 & 3.56 & 0.83 & 4.81 & 0.19 \\
\hline BDNF (ng/ml) & 13.58 & 7.77 & 22.56 & 19.95 & 9.31 & 29.45 & 10.97 & 2.50 & 14.14 & 0.008 \\
\hline
\end{tabular}

Of 78 patients with liver cirrhosis included in this study 43 patients were tested by psychometric hepatic encephalopathy score (PHES), inhibitory control test (ICT) and critical flicker frequency (CFF). The remaining 35 patients with liver cirrhosis while having serum BDNF measurement performed did not undergo complete MHE determination for different reasons, i.e. history of alcohol abuse and an ineligible MMSE test result.

The study protocol was approved by the Bioethics Committee of the Medical University in Bialystok (R-I-002/254/2015), and informed consent was obtained from each participant. The study was conducted in 2015-2017 at the Hepatology Department of the University Hospital in Białystok, Poland. The examination was carried out in two similar quiet rooms, usually between 15:00 and 18:00.

\section{Methods}

Minimal hepatic encephalopathy tests can be divided into psychometric, neurophysiological and neuroimaging tests. Leading scientific societies (European and American Associations for the Study of the Liver) recommend the use of the following tests as the most reliable: PHES as a gold standard, and one of the computerized tests: Continuous Reaction Time (CRT), ICT, SCAN, Stroop test, or neurophysiological CFF or electroencephalography (EEG) [24]. In this study MHE was diagnosed by PHES, ICT and CFF.

\section{Psychometric hepatic encephalopathy score (PHES)}

Psychometric hepatic encephalopathy score - the paper-and-pencil psychometric test battery is still the gold standard for MHE diagnosis [25]. PHES tests were performed in all 78 patients with liver cirrhosis. The PHES test was performed in accordance with generally accepted rules and comprised five tests: number connection tests A (NCT-A) and number connection tests B (NCT-B), serial dotting test (SDT), digit symbol test (DST) and line tracing test (LTT). We validated the PHES in a Polish population based on normative rules as previously described [26]. The results of the PHES were scored depending on the SD range in the control group. The results between \pm SD were scored 0 , between -1 and -2 SD were scored -1 , between -2 and -3 SD were scored -2 points, and more than $3 \mathrm{SD}$ were scored -3 . The final result was the sum of individual tests ranging between +5 and -15 points. MHE was recognized from a value of -5 and below $[27,28]$. The PHES test takes about 15 minutes. In our study, the cut-off for abnormal PHES was set at $\leq-5 \mathrm{SD}$.

The PHES test was validated against the control group. We examined 56 healthy volunteers [Polish population, 28 male, median age 54 (26-79) years, median years in education in 12 (8-23)]. The exclusion criteria were: illiteracy, visual or motor disturbance, neurological, psychiatric or liver disease, alcohol or any addictive substances abuse in the three months before the study. 


\section{Inhibitory control test (ICT)}

This test is a computer program measuring attention and inhibitory control. Bajaj et al. [29] proposed the ICT for the diagnosis of patients with liver cirrhosis and created a computer program. The test is freely available on the Internet. During the test, the patient is shown a sequence of letters on the computer screen, displayed in 500 millisecond intervals. The patient's task is to respond to a specific sequence of letters. In total, the program consists of 212 'targets' (X displayed after $\mathrm{Y}$ and vice versa) and 40 'lures' ( $\mathrm{X}$ after $\mathrm{X}$ and $\mathrm{Y}$ by $\mathrm{Y}$ ). The patient must press the button when $\mathrm{X}$ follows $\mathrm{Y}$ (or vice versa $\mathrm{Y}$ after $\mathrm{X}$ ) but refrain from responding when $\mathrm{X}$ is displayed after $\mathrm{X}$ or $\mathrm{Y}$ after $\mathrm{Y}$. The program automatically calculates the numbers and rates of correct and incorrect lure and target responses and response times. Before performing 5 test runs, the patient performs one training run [30]. The whole test takes about 15-20 minutes and does not require special training for the investigator. The free test was downloaded from the website: https://www.chronicliverdisease.org/disease_focus/ICT/. In the ICT study, the percentage of correct responses and the lures/target accuracy rate was taken into account in the diagnosis of MHE based on PHES.

\section{Critical flicker frequency (CFF)}

The examination was carried out using the "HEPAtonorm TM Analyzer (HE Flicker Diagnostics, nevoLAB GmbH, Germany)" in a quiet room. This analyser is small and portable. The set contains: goggles (the headset), a button, and a device with a screen that calculates the corresponding threshold frequencies and displays the results. The technique used in this test is the frequency measurement $(\mathrm{Hz})$. The screen of the "goggles" shows a light stimulus - a red spotlight, which is initially displayed at a frequency of $60 \mathrm{~Hz}$. At this high frequency, the light appears stable (does not move). The frequency is gradually reduced, giving the impression that the light is flickering/pulsating. The patient is asked to register (press the button) when this change occurs. Initially, the patient performs 5 training repetitions after discussing the rules. The test is repeated around 8 times and the device reports the mean CFF threshold [31-33]. In a study adapted using CFF for the diagnosis of MHE, with a cut-off of $39 \mathrm{~Hz}$, $100 \%$ efficacy was obtained in differentiating patients with symptoms of HE compared to the control group without cirrhosis and patients with cirrhosis HE 0 [34]. CFF is a reliable tool for diagnosing MHE, simple to administer, and can be performed by a non-special- ist. The results are independent of literacy and numeracy. The test can be performed in a short time (10-15 minutes) $[35,36]$. In this study we used a value of $39 \mathrm{~Hz}$ for MHE diagnosis.

\section{Routine blood measurements}

The liver biochemical parameters: alanine aminotransferase (ALT), aspartate aminotransferase (AST), $\gamma$-glutamyltranspeptidase (GGT); and lipids: total cholesterol, high-density lipoprotein (HDL), low-density lipoprotein (LDL), triglycerides (TC) were included. These parameters were measured using enzymatic methods and an automatic biochemical analytical platform COBAS INTEGRA 400 plus-blood count during physical examinations. The blood was collected on the day of the neurophysiological examination or up to 12 hours after its performance.

\section{Serum BDNF measurement}

Peripheral venous blood was collected from hospitalized patients in two tubes: a tube with clot activator to obtain serum, and a tube prefilled with EDTA K2 anticoagulant in order to separate the plasma. Blood samples were taken in the morning from fasting patients. The blood was centrifuged at $3000 x \mathrm{~g}$ for 15 minutes. Serum and plasma were collected and stored at $-80^{\circ} \mathrm{C}$ for further analysis. Serum BDNF was measured using an ELISA Total BDNF Quantikine ELISA Kit, R\&D, according to the manufacturer's recommendations. The minimum detectable level of human free BDNF is typically less than $20 \mathrm{pg} / \mathrm{ml}$. Serum BDNF concentration was validated against the control ( $n=40,32 \%$ female, median age 52 years) group of healthy volunteers without any liver disorders.

\section{Statistical analyses}

The normality of data distribution was verified with the Shapiro-Wilk test, and the homogeneity of variances with Levene's test. Non-parametric or parametric tests were used according to the type of data distribution. In the case of parametric distributions Student's $t$ test and the ANOVA test were used. Quantitative data were expressed as a median with a $25-75 \%$ confidence interval. Non-parametric tests were applied: the Kruskal-Wallis non-parametric ANOVA test, Mann-Whitney $U$-test for univariate comparisons, and the Spearman rank test for correlation analyses. Statistically significant $P$ values were $<0.05$. Statistical analyses were performed using the GraphPad Prism 8.4.2 (GraphPad Software, Inc. La Jolla, USA) and Statistica 13.1 software (StatSoft Tulsa, USA). 


\section{Results}

Seventy-eight patients with liver cirrhosis $(n=78)$ were enrolled in the current study. Of the entire study group, 43 patients were tested to assess the degree of MHE, of whom 29 had no MHE and 14 (33\%) were diagnosed with MHE with at least two of the abovementioned modalities. Patients with MHE had significantly lower serum albumin levels (2.7; 2.6-3.2 vs. 3.5; $3.1-3.7 \mathrm{mg} / \mathrm{dl}, p=0.006)$ and higher international normalized ratio (INR) $(1.34 ; 1.20-1.52$ vs. $1.17 ; 1.10-1.51$, $p=0.04$ ). They also had higher bilirubin and D-dimer levels, although the difference did not reach statistical significance (Table 1).

\section{BDNF concentration and biochemical liver parameters}

Serum concentration of BDNF was significantly lower in patients with cirrhosis $(13.6 ; 7.8-22.6 \mathrm{ng} / \mathrm{ml})$ compared to the control group $(33.0 ; 24.1-40.7 \mathrm{ng} / \mathrm{ml}$, $p<0.001$ ) (Fig. 1). BDNF in subjects with liver cirrhosis correlated positively with platelets (PLT) $(R=0.36$, $p=0.004)$, total protein $(R=0.31, p=0.04)$, and
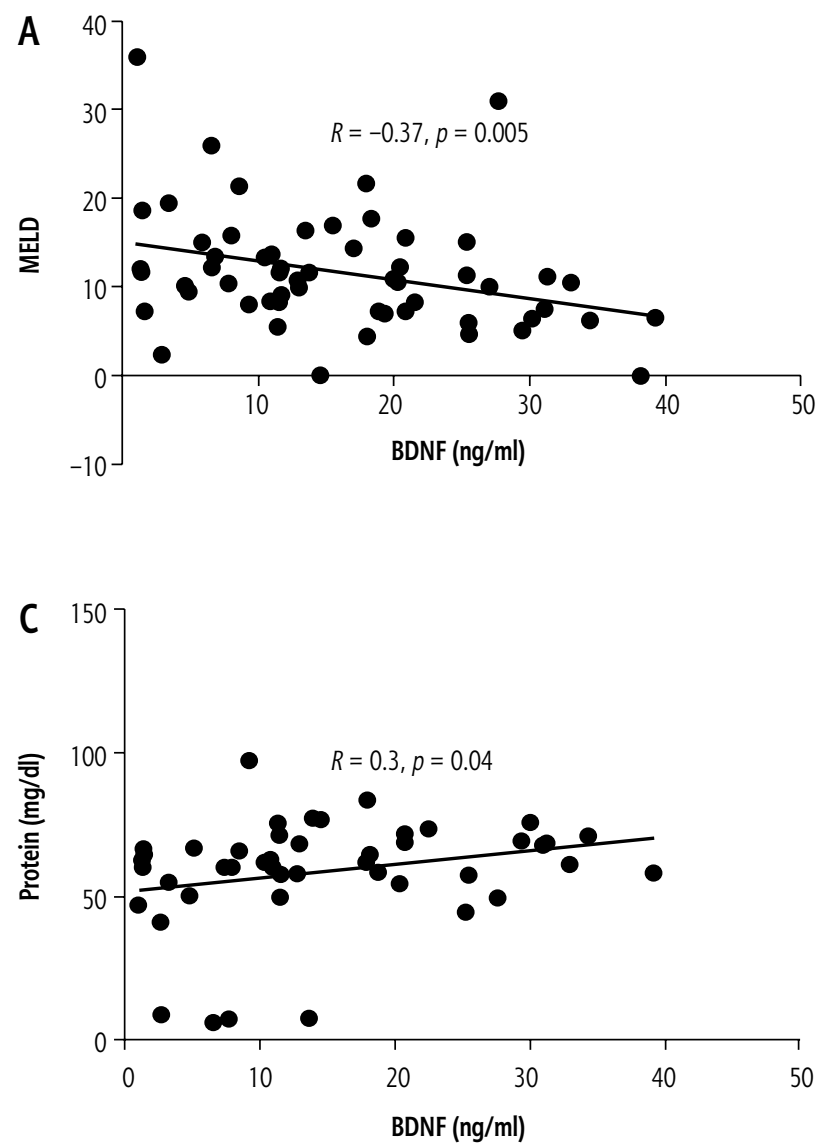

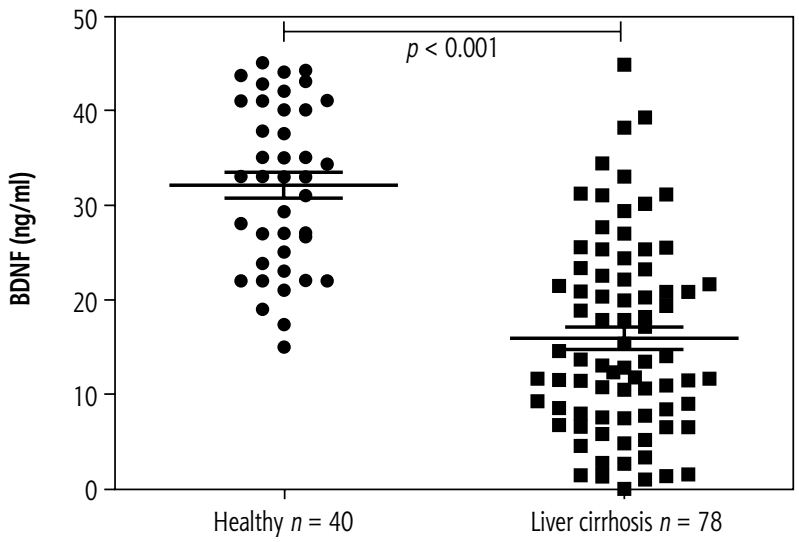

Fig. 1. Serum brain-derived neurotrophic factor (BDNF) concentration in cirrhotic patients compared to control group. Comparisons by use of MannWhitney $U$ test, $p<0.001$

negatively with bilirubin $(R=-0.35, p=0.005)$, INR $(R=-0.37, p=0.003)$, and model for end-stage liver disease (MELD) $(R=-0.35, p=0.005)$ (Fig. 2). No associations of serum BDNF with age, sex, body mass index (BMI), waist-hip ratio (WHR) or creatinine were recorded. Importantly, the lowest serum concentration of BDNF was noted in patients with MHE (11.0; $2.5-14.4 \mathrm{ng} / \mathrm{ml})$, which was significantly lower than in cirrhotics with-
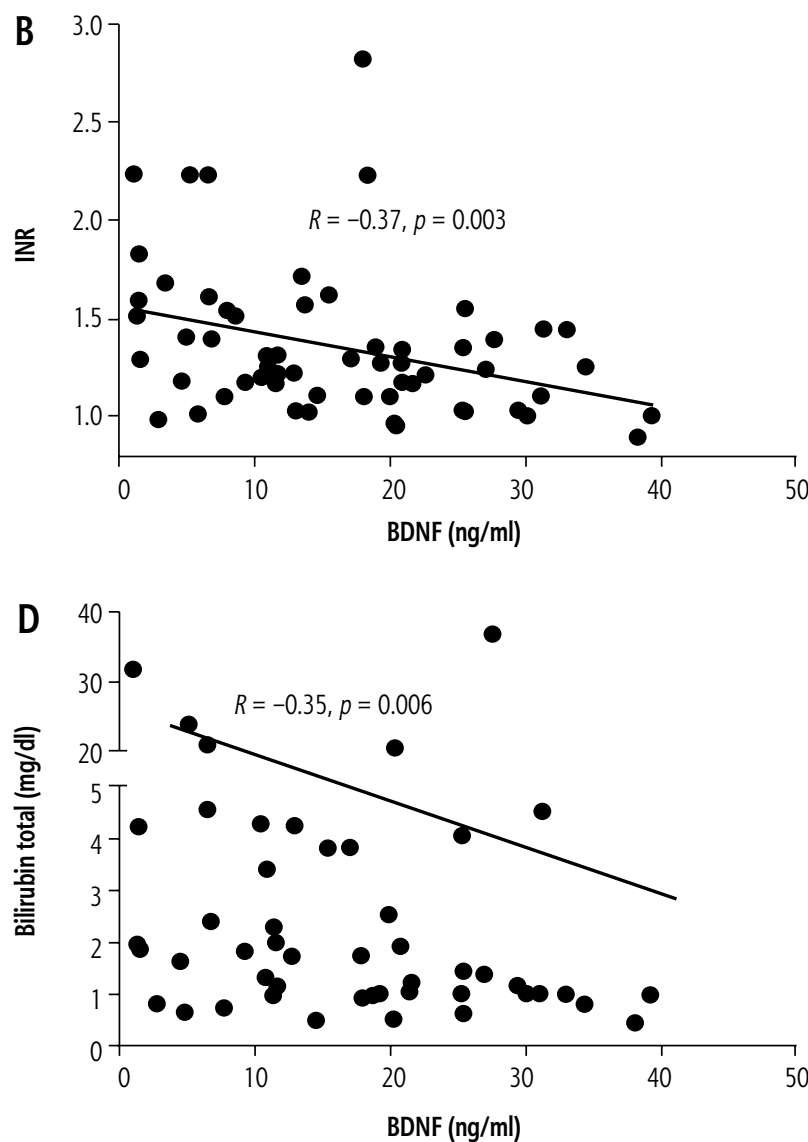

Fig. 2. Correlations between model for end-stage liver disease (MELD) score, international normalized ratio (INR) time, protein, and bilirubin with brain-derived neurotrophic factor (BDNF) activity in cirrhotic patients (Spearman rank test, $p<0.05$ ) 


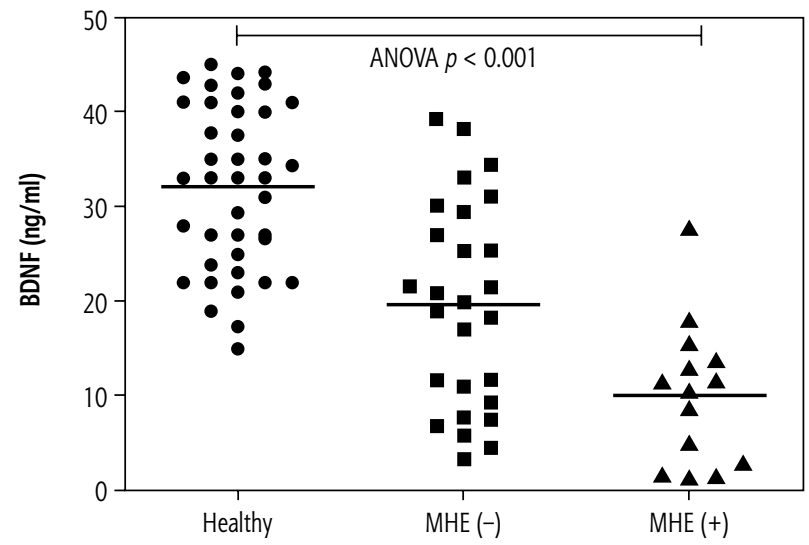

Fig. 3. Serum brain-derived neurotrophic factor (BDNF) concentration in patients with and without minimal hepatic encephalopathy (MHE) as compared to control group. Comparisons by use of Kruskal-Wallis ANOVA test, $p<0.001$

out MHE (19.9; 9.3-29.4 ng/ml, $p=0.008)$ and also in healthy subjects $(33.0 ; 24.1-40.7 \mathrm{ng} / \mathrm{ml}, p<0.001)$. In contrast, the difference in serum ammonia was not significant between MHE and non-MHE subjects, and serum ammonia did not correlate with BDNF $(r=0.01$, $p=0.95)$. Patients with self-reported sleep disturbances had significantly lower serum BDNF [13.0 (2.5-23.4) vs. $20.0(8.4-31.3) \mathrm{ng} / \mathrm{ml}, p=0.04]$.

\section{Discussion}

Early detection of patients with MHE may enable the identification of patients exposed to complications connected with liver cirrhosis. Interestingly, it has also been demonstrated that previous treatment with rifaximin or lactulose in patients with MHE significantly improves their quality of life [5]. Unfortunately, the diagnosis of MHE usually relies on a combination of neuropsychological tests, while robust biomarkers are lacking. BDNF is one of the neurotrophic factors which are involved in the differentiation and maturation of neurons in the nervous system. In addition, BDNF protein and its mRNA are found in most areas of the brain, such as the hippocampus, cortex, spinal cord, hypothalamus, midbrain, but also in the blood, making it a potentially good diagnostic marker [37]. Furthermore, BDNF is expressed in numerous tissues involved in energy production (adipose tissue, muscles and liver) [1].

Brain-derived neurotrophic factor has the ability to increase neurogenesis, which improves synaptic plasticity. It follows that a high level of this marker is conducive to maintaining normal cognitive functions. The relationship between serum BDNF concentration and different liver function classification in liver cirrhosis compared to patients with MHE has not been reported so far. In our study, we observed significantly lower
BDNF values both in patients with liver cirrhosis and in those with recognized MHE. Similarly to our study, decreased levels of BDNF were observed in a group of children with cirrhosis compared to the control group [1]. Moreover, low BDNF levels in patients with schizophrenia were associated with cognitive impairment, especially direct memory [38]. Importantly, a decreased value of $\mathrm{BDNF}$ is also accompanied by other neurodegenerative diseases, such as dementia, autism, multiple sclerosis, Parkinson's disease, Alzheimer's, and Huntington's disease [20-22, 37]. A study by Lim et al. [20] showed that the presence of Val66Met associated with BDNF polymorphism is responsible for synaptic excitation and integrity of neurons, which causes moderate memory loss associated with amyloid B and causes hippocampal atrophy in Alzheimer's disease [20]. In turn, the study by Zembron-Lacna et al. [38] proved the impact of physical activity and age on BDNF levels. In the study, young men had a significantly higher BDNF concentration than older men $(p<0.01)$. Active men, both older and younger, had higher BDNF levels compared to the group of inactive people. In active elderly men, BDNF concentration was $25 \%$ higher $(p<0.05)$ than in inactive young men. In addition, the study found a significant negative correlation of BDNF with atherogenic index (TC/HDL), high-sensitivity C-reactive protein (hsCRP) and oxLDL concentration [38]. In our research with MHE patients we also noted a significant decrease in BDNF in the group with recognized MHE compared to healthy controls. Furthermore, BDNF concentration distinguished patients with liver cirrhosis vs. the control group $(p<0.0001)$. In the whole study group, patients with cirrhosis as well as those diagnosed with MHE had bilirubin levels above the upper limit, as is common in patients with severe liver damage. Large changes in the liver are confirmed by significantly higher ALT, AST and GGT in all our patients, compared to the control group. It should be remembered that BDNF expression reduces both stress and inflammation [1]. Cirrhosis-associated combination with cognitive changes in the brain can lead to a decrease in BDNF values. Furthermore, in our study the lowest BDNF values were observed in patients with diagnosed MHE, median $11.0 \mathrm{ng} / \mathrm{dl}$ (2.5-14.4), slightly higher in patients without MHE, median $19.9 \mathrm{ng} / \mathrm{ml}(9.3-29.4)$, and the highest in the control group, median $33.0 \mathrm{ng} / \mathrm{ml}$ (24.1-40.7), which allowed us to differentiate between statistically ill patients from the healthy ones $(p<0.001)$. BDNF is involved in several complex processes, and its expression has a protective role by reducing stress and inflammation [25]. Interestingly, MHE severity is not closely related to the clinical stage of cirrhosis or serum ammo- 
nia levels [39]. In our study, an increase in ammonia activity above the reference value was observed in the majority of subjects with liver cirrhosis, but it did not distinguish patients with diagnosed MHE. We noted even lower values in the MHE (+) group compared to MHE (-). However, the diagnosis of MHE correlated with serum albumins and INR. Serum BDNF levels were also associated with Child-Pugh liver function classes in patients with HBV-induced cirrhosis [40].

Recent reports suggest that cognitive disorders in patients with cirrhosis may not be fully reversible after a manifestation of apparent HE. Görg et al. [17] studied the influence of prolonged exposure to ammonia on the functions of astrocytes by analysing the biomarker expression level, i.e. ammonia concentration in rat astrocyte culture. The concentration of, as well as time of exposure to, $\mathrm{NH}_{4}$ inhibited the proliferation of astrocytes to $45 \%(5 \mathrm{mmol} / \mathrm{l}, 72 \mathrm{~h})$ and strongly increased the activity of $\beta$-galactosidase compared to the control group. It is known that $\mathrm{NH}_{4} \mathrm{Cl}(5 \mathrm{mmol} / \mathrm{l})$ inhibits the astrocyte proliferation induced by epidermal growth factor and BDNF. BDNF mediated changes in astrocytes by downregulating the number of receptors for epidermal growth factor and truncated tyrosine kinase receptor B. Tumour protein p53, the GADD45a gene, and p21 are responsible for inhibition of astrocyte proliferation by ammonia. Increased expression of mRNA p21, p53, and GADD45a was observed in a study of brain tissue of cirrhosis patients with developed $\mathrm{HE}$, but it was not demonstrated in cirrhosis patients without any signs of MHE [17]. The administration of nerve growth factor in mice showed that it may reverse cholinergic neuron atrophy in the basal part of the cerebrum, and improve behavioural deficits [41]. Negative correlations of INR, MELD and bilirubin with BDNF concentration obtained in our experiment might also link the degree of neural degeneration and liver damage. Serum protein concentration is the result of protein production in the liver and protein loss by the kidneys and digestive tract. Positive correlation of protein with BDNF occurs most frequently in the group of patients with the lowest degree of liver damage and MHE (-), which suggests less liver damage, i.e. persistent production of protein, but also smaller changes in cognitive processes in the brain, as evidenced by higher BDNF values. A relationship between the loss of BDNF activity and proteins was also observed in rat studies [39]. It is known that cirrhosis contributes to the development of serious psychological and social problems. Difficulties with depression and impaired memory may contribute to the deterioration of health $[3,39]$. Therefore, testing BDNF as a marker of early brain changes from those irreversible as in HE seems justified. However, it is suggested that BDNF in patients with liver cirrhosis acts not only as a neurotrophic marker, but also as an important molecule associated with the severity of inflammation and fibrosis [3]. In addition, clinical studies confirm the use of serum BDNF as a marker of the therapeutic effectiveness of antidepressants. BDNF transcription is extremely sensitive to regulation by neuronal activity. Interesting studies in rats showed that treatment with BDNF antisense oligonucleotides led to an improvement in mental state but more importantly a recall of spatial memory [39]. It is worth emphasizing that BDNF is involved in the regulation of cognitive functions. Stress and inflammatory processes lead to changes in the level of BDNF. High concentrations of glucocorticoid hormones and proinflammatory cytokines have been observed in response to stress, which resulted from reduced levels of BDNF and changes in the function of hippocampal neurons (such as dendritic atrophy, neurodegeneration and decreased neurogenesis) [19, 37, 39].

The present study has limitations resulting from the small study population. It concerns both the number of patients with cirrhosis and the number of people in the control group, in which the BDNF concentration was measured. Undoubtedly, the presented hypotheses require further research in larger groups of patients with MHE.

In summary, this study shows the reduced serum BDNF concentration in patients with cirrhosis, reflecting the degree of inflammation of the liver, but also liver disease progression. In addition, reduced cognitive function in MHE correlates with decreased BDNF expression. This study also suggests the possible clinical utility of BDNF determination in differentiating patients with cirrhosis from those with MHE symptoms. Finally, our results could be a starting point to study the potential therapeutic significance of BDNF to prevent cognitive deficits observed in patients with MHE and cirrhosis.

\section{Disclosure}

The authors declare no conflict of interest.

\section{References}

1. Wilasco MIA, Uribe-Cruz C, Santetti D, et al. Brain-derived neurotrophic factor in children and adolescents with cirrhosis due to biliary atresia. Ann Nutr Metab 2016; 69: 1-8.

2. Avila L, Weinstein AA, Estep JM, et al. Cytokine balance is restored as patient-reported outcomes improve in patients recovering from chronic hepatitis C. Liver Int 2019; 39: 1631-1640.

3. Xu H, Zhou Y, Ko F, et al. Female gender and gastrointestinal symptoms, not brain-derived neurotrophic factor, are associat- 
ed with depression and anxiety in cirrhosis. Hepatol Res 2017; 47: E64-E73.

4. Stawicka A, Zbrzeźniak J, Świderska A, et al. Clinical importance and diagnostic methods of minimal hepatic encephalopathy. Pol Merkur Lekarski 2016; 40: 117-121.

5. Ridola L, Nardelli S, Gioia S, et al. Quality of life in patients with minimal hepatic encephalopathy. World J Gastroenterol 2018; 24: 5446-5453.

6. Reynolds AS, Brush B, Schiano TD, et al. Neurological monitoring in acute liver failure. hepatology. John Wiley and Sons Inc. 2019; 1830-1835.

7. Lima LCD, Miranda AS, Ferreira RN, et al. Hepatic encephalopathy: Lessons from preclinical studies. World J Hepatol 2019; 11: 173-185.

8. Sato T, Endo K, Kakisaka K, et al. Decreased mean kurtosis in the putamen is a diagnostic feature of minimal hepatic encephalopathy in patients with cirrhosis. Intern Med 2019; 58: 1217-1224.

9. Kooka Y, Sawara K, Endo R, et al. Brain metabolism in minimal hepatic encephalopathy assessed by 3.0-Tesla magnetic resonance spectroscopy. Hepatol Res 2016; 46: 269-276.

10. Nardelli S, Gioia S, Faccioli J, et al. Sarcopenia and cognitive impairment in liver cirrhosis: A viewpoint on the clinical impact of minimal hepatic encephalopathy. World J Gastroenterol 2019; 25: 5257-5265.

11. Blei AT, Ferenci P, Lockwood A, et al. Hepatic encephalopathy - Definition, nomenclature, diagnosis, and quantification: Final report of the Working Party at the 11th World Congresses of Gastroenterology, Vienna, 1998. Hepatology 2002; 35: 716-721.

12. Ortiz M, Jacas C, Córdoba J. Minimal hepatic encephalopathy: Diagnosis, clinical significance and recommendations. J Hepatol 2005; 42 Suppl: S45-53.

13. Bajaj JS, Hafeezullah M, Hoffmann RG, et al. Minimal hepatic encephalopathy: A vehicle for accidents and traffic violations. Am J Gastroenterol 2007; 102: 1903-1909.

14. Bajaj JS, Ananthakrishnan AN, McGinley EL, et al. Deleterious effect of cirrhosis on outcomes after motor vehicle crashes using the nationwide inpatient sample. Am J Gastroenterol 2008; 103 : 1674-1681.

15. Bamidele OF, Olokoba AB, Bojuwoye MO, et al. Prevalence of minimal hepatic encephalopathy among patients with chronic liver disease in Ilorin, Nigeria. Ghana Med J 2019; 53: 299-303.

16. Sidhu SS, Goyal O, Parker RA, et al. Rifaximin vs. lactulose in treatment of minimal hepatic encephalopathy. Liver Int 2016; 36: 378-385.

17. Görg B, Karababa A, Häussinger D. Hepatic encephalopathy and astrocyte senescence. J Clin Exp Hepatol 2018; 8: 294-300.

18. Häussinger D, Sies H. Hepatic encephalopathy: clinical aspects and pathogenetic concept. Arch Biochem Biophys 2013; 536: 97-100.

19. Maqsood R, Stone TW. The gut-brain axis, BDNF, NMDA and CNS disorders. Neurochem Res 2016; 41: 2819-2835.

20. Lim YY, Hassenstab J, Cruchaga C, et al. BDNF Val66Met moderates memory impairment, hippocampal function and tau in preclinical autosomal dominant Alzheimer's disease. Brain 2016; 139: 2766-2777.

21. Buchman AS, Yu L, Boyle PA, et al. Higher brain BDNF gene expression is associated with slower cognitive decline in older adults. Neurology 2016; 86: 735-741.

22. Libman-Sokołowska M, Drozdowicz E, Nasierowski T. BDNF jako biomarker w przebiegu i leczeniu schizofrenii BDNF as a biomarker in the course and treatment of schizophrenia. Psychiatr Pol 2015; 49: 1149-1158.
23. Korte M, Carroll P, Wolf E, et al. Hippocampal long-term potentiation is impaired in mice lacking brain-derived neurotrophic factor. Proc Natl Acad Sci U S A 1995; 92: 8856-8860.

24. Vilstrup H, Amodio P, Bajaj J, et al. Hepatic encephalopathy in chronic liver disease: 2014 Practice Guideline by the American Association for the Study of Liver Diseases and the European Association for the Study of the Liver. Hepatology 2014; 60: 715-735.

25. Luo M, Ma P, Li L, et al. Advances in psychometric tests for screening minimal hepatic encephalopathy: From paper-and-pencil to computer-aided assessment. Turkish J Gastroenterol 2019; 30: 398-407.

26. Stawicka A, Jaroszewicz J, Zbrzeźniak J, et al. Clinical usefulness of the inhibitory control test (ICT) in the diagnosis of minimal hepatic encephalopathy. Int J Environ Res Public Health 2020; 17: 3645 .

27. Wunsch E, Koziarska D, Kotarska K, et al. Normalization of the psychometric hepatic encephalopathy score in Polish population. A prospective, quantified electroencephalography study. Liver Int 2013; 33: 1332-1340.

28. Weissenborn K. PHES: One label, different goods?! J Hepatol 2008; 49: 308-312.

29. Bajaj JS, Saeian K, Verber MD, et al. Inhibitory control test is a simple method to diagnose minimal hepatic encephalopathy and predict development of overt hepatic encephalopathy. Am J Gastroenterol 2007; 102: 754-760.

30. Taneja S, Dhiman RK, Khatri A, et al. Inhibitory control test for the detection of minimal hepatic encephalopathy in patients with cirrhosis of liver. J Clin Exp Hepatol 2012; 2: 306-314.

31. Manual. HEPAtonorm TM Analyzer (HE Flicker Diagnostics, Düsseldorf, GermanynevoLAB GmbH, Germany).

32. Romero-Gómez M, Córdoba J, Jover R, et al. Value of the critical flicker frequency in patients with minimal hepatic encephalopathy. Hepatology 2007; 45: 879-885.

33. Barone M, Shahini E, Iannone A, et al. Critical flicker frequency test predicts overt hepatic encephalopathy and survival in patients with liver cirrhosis. Dig Liver Dis 2018; 50: 496-500.

34. Häussinger D, Wettstein M, Kircheis G, et al. Critical flicker frequency for quantification of low-grade hepatic encephalopathy. Hepatology 2002; 35: 357-366.

35. Torlot FJ, McPhail MJW, Taylor-Robinson SD. Meta-analysis: The diagnostic accuracy of critical flicker frequency in minimal hepatic encephalopathy. Aliment Pharmacol Ther 2013; 37: 527-536.

36. Coşkun BDO, Ozen M. Critical flicker frequency test for diagnosing minimal hepatic encephalopathy in patients with cirrhosis. Turkish J Gastroenterol 2017; 28: 191-196.

37. Bathina S, Das UN. Brain-derived neurotrophic factor and its clinical implications. Arch Med Sci 2015; 11: 1164-1178.

38. Zembron-Lacny A, Dziubek W, Rynkiewicz M, et al. Peripheral brain-derived neurotrophic factor is related to cardiovascular risk factors in active and inactive elderly men. Brazilian J Med Biol Res 2016; 49: 1-8.

39. Dhanda S, Gupta S, Halder A, et al. Systemic inflammation without gliosis mediates cognitive deficits through impaired BDNF expression in bile duct ligation model of hepatic encephalopathy. Brain Behav Immun 2018; 70: 214-232.

40. Shu HC, Hu J, Jiang XB, et al. BDNF gene polymorphism and serum level correlate with liver function in patients with hepatitis B-induced cirrhosis. Int J Clin Exp Pathol 2019; 12: 2368-2380.

41. Chen KS, Nishimura MC, Armanini MP, et al. Disruption of a single allele of the nerve growth factor gene results in atrophy of basal forebrain cholinergic neurons and memory deficits. J Neurosci 1997; 17: 7288-7296. 